\title{
Prognostic significance of the postoperative level and recovery rate of serum albumin in patients with curatively resected pancreatic ductal adenocarcinoma
}

\author{
YUTAKA NAKANO $^{1}$, MINORU KITAGO ${ }^{1}$, MASAHIRO SHINODA $^{1}$, HIROSHI YAGI $^{1}$, YUTA ABE $^{1}$, \\ KIMINORI TAKANO $^{1}$, GO OSHIMA ${ }^{1}$, AYANO TAKEUCH ${ }^{2}$, YUTAKA ENDO ${ }^{1}$ and YUKO KITAGAWA ${ }^{1}$ \\ Departments of ${ }^{1}$ Surgery and ${ }^{2}$ Preventive Medicine and Public Health, Keio University School of Medicine, \\ Tokyo 160-8582, Japan
}

Received October 5, 2018; Accepted May 30, 2019

DOI: $10.3892 /$ mco.2019.1883

\begin{abstract}
The aim of the present study was to investigate the clinicopathological features and prognostic factors associated with pre- and postoperative serum albumin levels in patients with curatively resected pancreatic ductal adenocarcinoma (PDAC). To achieve this, the data of patients who underwent pancreatectomy for PDAC between January 1995 and March 2016 were retrospectively reviewed, and the pre- and postoperative serum albumin levels at postoperative months (POMs) 3, 6, and 12 were evaluated. The serum albumin recovery rate was also investigated. A total of 196 patients were enrolled in the present study. In the multivariate Cox regression analysis, lymph node metastasis [hazard ratio (HR): $1.65 ; \mathrm{P}=0.022$ ], serum albumin level at POM 12 ( $\geq 3.9 \mathrm{~g} / \mathrm{dl}$; HR: 0.60; P=0.017), and serum albumin recovery rate at POM $12(\geq 1.00$; HR: $0.60 ; \mathrm{P}=0.017)$ were independent prognostic factors for disease-free survival. Lymph node metastasis (HR: $1.79 ; \mathrm{P}=0.013$ ) and serum albumin level at POM 12 ( $\geq 3.9 \mathrm{~g} / \mathrm{dl})$ (HR: 0.60; $\mathrm{P}=0.033)$ were independent prognostic factors for overall survival. These results indicated that the postoperative level and recovery rate of serum albumin are potential biomarkers for predicting the prognosis of patients with curatively resected PDAC. However, further studies are required in order to investigate the survival benefit of increasing postoperative serum albumin levels in these patients.
\end{abstract}

\section{Introduction}

Pancreatic ductal adenocarcinoma (PDAC) represents one of the most lethal and aggressive forms of cancer. The 5 year survival rate of patients after surgical resection for PDAC

Correspondence to: Dr Minoru Kitago, Department of Surgery, Keio University School of Medicine, 35 Shinanomachi, Shinjuku-ku, Tokyo 160-8582, Japan

E-mail: dragonpegasus@keio.jp

Key words: biomarker, pancreatic cancer, prognosis, serum albumin, survival remains poor. In Japan, the 5 year survival rate is $18.8 \%$ (1). In the United States and Europe, it is $20.0 \%$ (2). However, the survival rate of patients with PDAC has increased due to advancements in multimodal therapies $(3,4)$.

Serum albumin is the most abundant blood plasma protein in humans and is produced by the liver (5). Low serum albumin levels may be caused by liver disease (5), systematic inflammation (6), the disease state (7), malnutrition (8), and sarcopenia (9). Several studies have identified clinicopathological prognostic factors associated with serum albumin levels in patients with PDAC, including the C-reactive protein (CRP)-to-albumin ratio (10), prognostic nutritional index (PNI) (11), and modified Glasgow Prognostic Score (mGPS) (12). However, no studies have reported that postoperative serum albumin levels predict survival in patients with PDAC.

Recently, important technological advances have facilitated the identification of biomarkers for PDAC. For example, DNA from tumor tissue and serum from PDAC patients have potential clinical utility as biomarkers for monitoring treatment response and predicting survival (13). We previously reported the pre- and postoperative clinicopathological characteristics of patients with PDAC who survived for $>5$ years after curative resection (1995-2011) (14). We also reported that preoperative serum albumin levels may be a predictive biomarker for achieving 5 year survival in patients with curatively resected PDAC. Thus, in the present study, we investigated prognostic factors associated with serum albumin, hypothesizing that the postoperative level and/or recovery rate of serum albumin is associated with survival after pancreatectomy.

\section{Materials and methods}

Patients. Data of patients who underwent intended curative pancreatectomy for PDAC at our institution between January 1995 and March 2016 were retrospectively reviewed. All patients had histologically confirmed PDAC. Patients were excluded if they had liver cirrhosis, R2 resection, or died within 12 months of pancreatectomy. We conducted a retrospective observational study using the 'opt-out' method of our hospital. The study was approved by the Ethics Committee of Keio University School of Medicine. 
Preoperative assessment. Demographic and clinical variables included age, sex, surgical procedure, neoadjuvant chemoradiotherapy, operative time, blood loss, and postoperative complications (e.g., pancreatic fistula, intraabdominal bleeding, delayed gastric emptying, and fluid collection), which were evaluated using the Clavien-Dindo classification. Preoperative laboratory data, including the CRP-to-albumin ratio, PNI, and mGPS, were also collected.

Since 2003, at our hospital, neoadjuvant chemoradiotherapy has been administered to patients who were diagnosed with T3/T4 disease according to the Union for International Cancer Control Tumor-Node-Metastasis Classification of Malignant Tumors ( $7^{\text {th }}$ edition) $(15)$.

Surgery and pathology. Surgical procedures included pancreaticoduodenectomy, distal pancreatectomy, and total pancreatectomy. D2 lymph node dissection was performed in all patients. Pathological staging was determined according to the Union for International Cancer Control Tumor-Node-Metastasis Classification of Malignant Tumors (7th edition) (15). R0 resections were defined as cases without gross or microscopic evidence of residual disease. R1 resections had microscopically positive margins, and $\mathrm{R} 2$ resections still contained some gross tumor matter. Pathological features associated with prognosis included histologically assessed tumor size; distal bile duct, duodenal, serosal, retropancreatic tissue, portal venous or arterial system, or extrapancreatic nerve plexus invasion; other organ invasion; lymph node metastasis (LNM); and lymphatic, venous, or intrapancreatic neural infiltration (16).

Perioperative portal vein infusion chemotherapy. Since 1986, perioperative portal vein infusion chemotherapy has been performed as a standard treatment at our hospital to prevent liver recurrence and improve survival in patients with PDAC who have undergone potentially curative resection $(17,18)$.

Pre- and postoperative serum albumin. Preoperative serum albumin levels were measured before pancreatectomy. Postoperative serum albumin levels were evaluated at postoperative months (POMs) 3, 6, and 12. The postoperative serum albumin recovery rate was defined as the serum albumin level at POMs 3,6 , or $12(\mathrm{~g} / \mathrm{dl})$ divided by the preoperative serum albumin level $(\mathrm{g} / \mathrm{dl})$.

Follow-up. Patients were followed-up at POMs 1, 3, 6, and 12 Patients were also subject to semiannual reviews. Clinical examinations, laboratory investigations, and abdominal computed tomography scans (to detect tumor recurrence) were performed. Patients were follow-up until death or March 2017.

Statistical analyses. Survival curves were plotted using the Kaplan-Meier method and compared using the log-rank test. Disease-free survival (DFS) was defined as the time interval between the date of surgery and the date of recurrence or last follow-up. Overall survival (OS) was defined as the time interval between the date of surgery and the date of death or last follow-up. Categorical variables were compared using the chi-square or Fisher's exact test. Cox proportional hazards regression models were used to determine independent prognostic factors between January 1995 and March 2016. The optimum cutoff value for postoperative serum albumin levels at POMs 3, 6, and 12 was $3.9 \mathrm{~g} / \mathrm{dl}$, according to our previous report (14). In the present study, the optimum cutoff values for albumin on POM 3, POM 6 and POM 12 were also calculated beforehand and the results were 3.6, 3.7, $3.7 \mathrm{~g} / \mathrm{dl}$ respectively (Table SI). The results were quite similar between using unified cutoff value $(3.9 \mathrm{~g} / \mathrm{dl})$ and optimum cutoff values, therefore the unified cutoff value of $3.9 \mathrm{~g} / \mathrm{dl}$ was used. The association between early recurrence (within 1 year) and the postoperative administration of adjuvant chemotherapy was analyzed using Spearman's rank correlation coefficient. All statistical analyses were conducted using Statistical Package for the Social Sciences for Macintosh (software v.23.0; IBM Corp., Armonk, NY, USA). P<0.05 was considered to indicate a statistically significant difference.

\section{Results}

Patient characteristics and surgical procedures. In total, 247 patients underwent curative pancreatectomy at our hospital between January 1995 and March 2016. Fifty-one patients died within 12 months of surgery. Therefore, 196 patients were enrolled. The patient characteristics are summarized in Table I. Among the 51 patients who died within 12 months of surgery, 39 (76.5\%) of deaths were due to recurrence of PDAC, $8(15.7 \%)$ were due to other diseases, and $4(7.8 \%)$ were from unknown causes. Recurrent lesions in 39 patients were associated with peritoneal dissemination, liver metastasis, and local recurrence. There were no specific factors associated with poor survival, but in patients that died within 12 months of surgery because of PDAC, peritoneal dissemination and liver metastasis were the main lesions of recurrence.

Postoperative albumin levels, albumin recovery rate, and survival. Kaplan-Meier survival curves of all patients are shown in Fig. 1A and B. Patients with postoperative serum albumin levels of $\geq 3.9 \mathrm{~g} / \mathrm{dl}$ at POMs 3 and 6 did not exhibit a significantly longer DFS or OS. However, patients with postoperative serum albumin levels of $\geq 3.9 \mathrm{~g} / \mathrm{dl}$ at POM 12 did exhibit a significantly longer DFS and OS (both $\mathrm{P}<0.001$; Fig. 2A and B). Patients with a postoperative serum albumin recovery rate of $\geq 1.00$ at $\mathrm{POM} 3$ had a significantly longer DFS $(\mathrm{P}=0.047)$. Patients with a postoperative serum albumin recovery rate of $\geq 1.00$ at $\mathrm{POM} 12$ had a significantly longer DFS and $\mathrm{OS}(\mathrm{P}<0.001$ and $\mathrm{P}=0.001$, respectively; Fig. $3 \mathrm{~A}$ and $\mathrm{B})$.

The results of the univariate and multivariate analyses of DFS and OS are shown in Table II. In the univariate analysis, the CRP-to-albumin ratio, PNI, and mGPS were not significant prognostic factors for DFS or OS. The postoperative albumin level and albumin recovery rate at POMs 3 and 6 were also not significant prognostic factors for DFS or OS. However, the postoperative albumin level and albumin recovery rate at POM 12 were significant prognostic factors for DFS. Lymph node involvement, venous involvement, and LNM were also significant prognostic factors for DFS. Neural involvement and LNM were significant prognostic factors for OS. Multivariate analysis confirmed venous involvement (HR: $0.75 ; \mathrm{P}=0.030$ ), LNM (HR: $1.65 ; \mathrm{P}=0.022$ ), serum albumin level at POM 12 ( $\geq 3.9 \mathrm{~g} / \mathrm{dl}$ ) (HR: 0.60; $\mathrm{P}=0.017$ ), and serum albumin recovery 
Table I. Patient characteristics.

\begin{tabular}{lc}
\hline Characteristics & Patients $(n=196)$ \\
\hline Age (years), median (range) & $68(38-87)$ \\
Sex, $n(\%)$ & \\
M & $130(66.3)$ \\
F & $66(33.7)$ \\
Surgical procedure, $n(\%)$ & \\
PD & $106(54.1)$ \\
DP & $80(40.8)$ \\
TP & $10(5.1)$ \\
NACRT, $n(\%)$ & $37(18.9)$ \\
Pathological stage, $n(\%)^{\mathrm{a}}$ & \\
IA & $11(5.6)$ \\
IB & $9(4.6)$ \\
IIA & $51(26.0)$ \\
IIB & $124(63.3)$ \\
III & $1(0.5)$ \\
IV & $0(0.0)$ \\
Perioperative PVI chemotherapy, $n(\%)$ & $121(62.7)$ \\
Adjuvant chemotherapy, $n(\%)^{\mathrm{b}}$ & $132(67.3)$ \\
Resection status, $n(\%)$ & \\
R0 & $150(76.5)$ \\
R1 & $46(23.5)$ \\
\hline
\end{tabular}

${ }^{a}$ UICC TNM Classification of Malignant Tumors (7th edition). ${ }^{\mathrm{b}} \mathrm{S}-1$, gemcitabine, mitomycin C, or 5-FU. 5-FU, 5-fluorouracil; F, female; DP, distal pancreatectomy; M, male; NACRT, neoadjuvant chemoradiotherapy; PD, pancreatoduodenectomy; PVI, portal vein infusion; S-1, tegafur/gimeracil/oteracil; TNM, Tumor-Node-Metastasis; TP, total pancreatectomy; UICC, Union for International Cancer Control.

rate at $\mathrm{POM} 12(\geq 1.00)$ (HR: $0.60 ; \mathrm{P}=0.017)$ to be independent prognostic factors for DFS. LNM (HR: 1.79; $\mathrm{P}=0.013)$ and serum albumin level at POM 12 ( $\geq 3.9 \mathrm{~g} / \mathrm{dl}$ ) (HR: 0.60; $\mathrm{P}=0.033$ ) were independent prognostic factors for OS (Table II).

Adjuvant chemotherapy and early recurrence. Patients with a serum albumin level of $<3.9 \mathrm{~g} / \mathrm{dl}$ at POMs 6 and $12 \mathrm{did}$ not receive postoperative adjuvant chemotherapy compared to those with a serum albumin level of $\geq 3.9 \mathrm{~g} / \mathrm{dl}$ at POMs 6 and $12(\mathrm{P}=0.038$ and $\mathrm{P}=0.009$, respectively). These patients also exhibited significantly early recurrence (within 1 year) $(\mathrm{P}=0.029$ and $\mathrm{P}=0.001$, respectively).

\section{Discussion}

We investigated the prognostic significance of pre- and postoperative serum albumin levels and the serum albumin recovery rate for predicting survival after pancreatectomy in patients with PDAC. Our results showed that serum albumin levels at POM 12 were an independent prognostic factor for both DFS and OS, and that the serum albumin recovery rate at POM 12 was an independent prognostic factor for DFS.
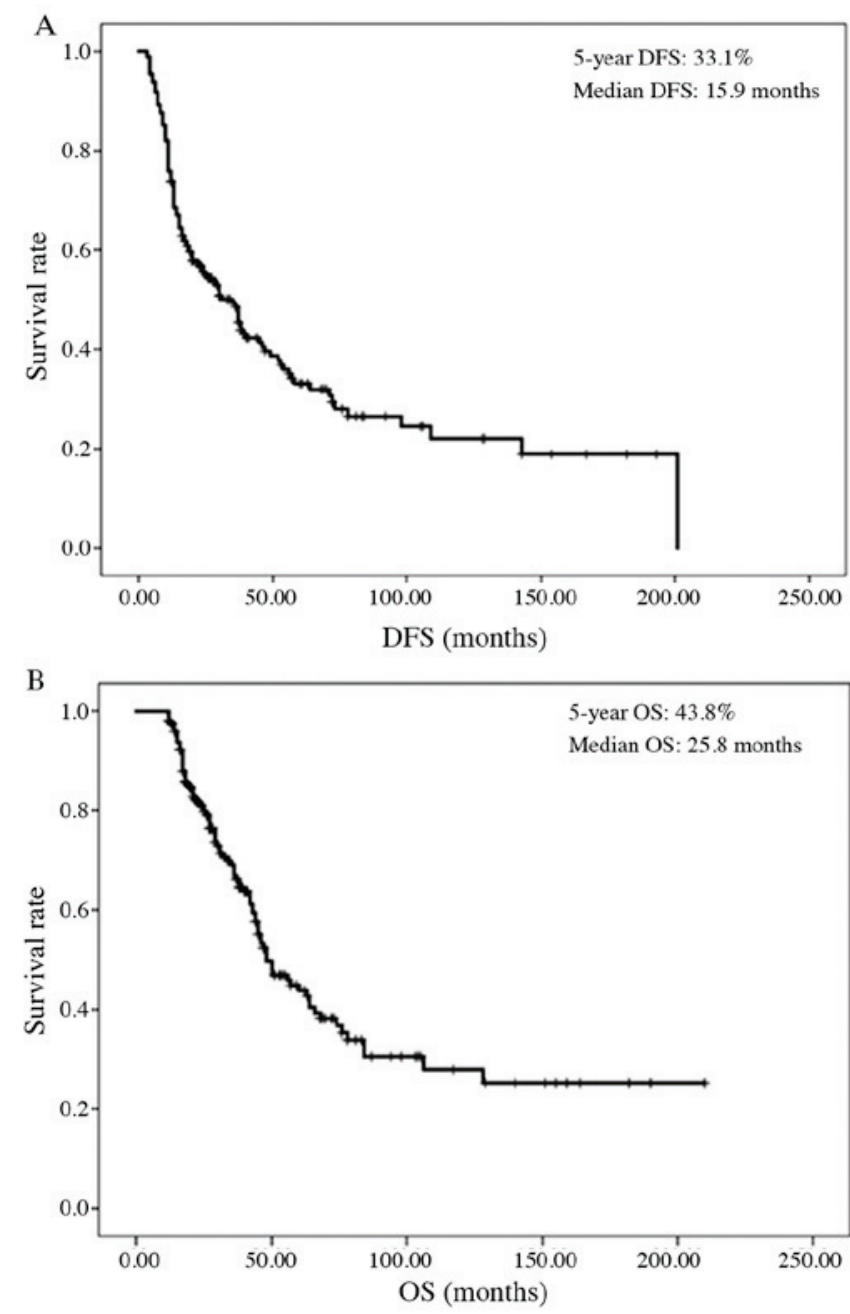

Figure 1. Kaplan-Meier curves of (A) postoperative DFS and (B) postoperative OS for all patients. DFS, disease-free survival; OS, overall survival.

Compared with other prognostic factors or scores (e.g., the CRP-to-albumin ratio, PNI, and mGPS), serum albumin levels may be straightforward because they do not require any parameters. Furthermore, patients with a postoperative serum albumin level of $\geq 3.9 \mathrm{~g} / \mathrm{dl}$ at POM 12 or a postoperative serum albumin recovery rate of $\geq 1.00$ at POM 12 exhibited a significantly longer survival.

Albumin is a constitutive hepatic protein that accounts for $50.0 \%$ of serum proteins produced by the liver and is regulated by various factors (5). Low serum albumin levels are associated with advanced PDAC (7), malnutrition (8), and sarcopenia (9). Obstructive cholangitis or pancreatitis is usually improved after surgery. Thus, in contrast to preoperative serum albumin levels, low postoperative serum albumin levels may be associated with cancer recurrence or inflammation, especially postoperative serum albumin levels at POM 12. Serum albumin levels have been associated with survival in oncologic patients in locally or metastatic types of cancer, and a low serum albumin may be due to a sustained systemic inflammatory response from an aggressive metabolically active tumor (5). Preoperative and postoperative ongoing inflammation leads to the production of acute phase proteins, and if this process is prolonged or aggressive, such as in advanced PDAC, it can lead to significant depletion of protein reserves and a 

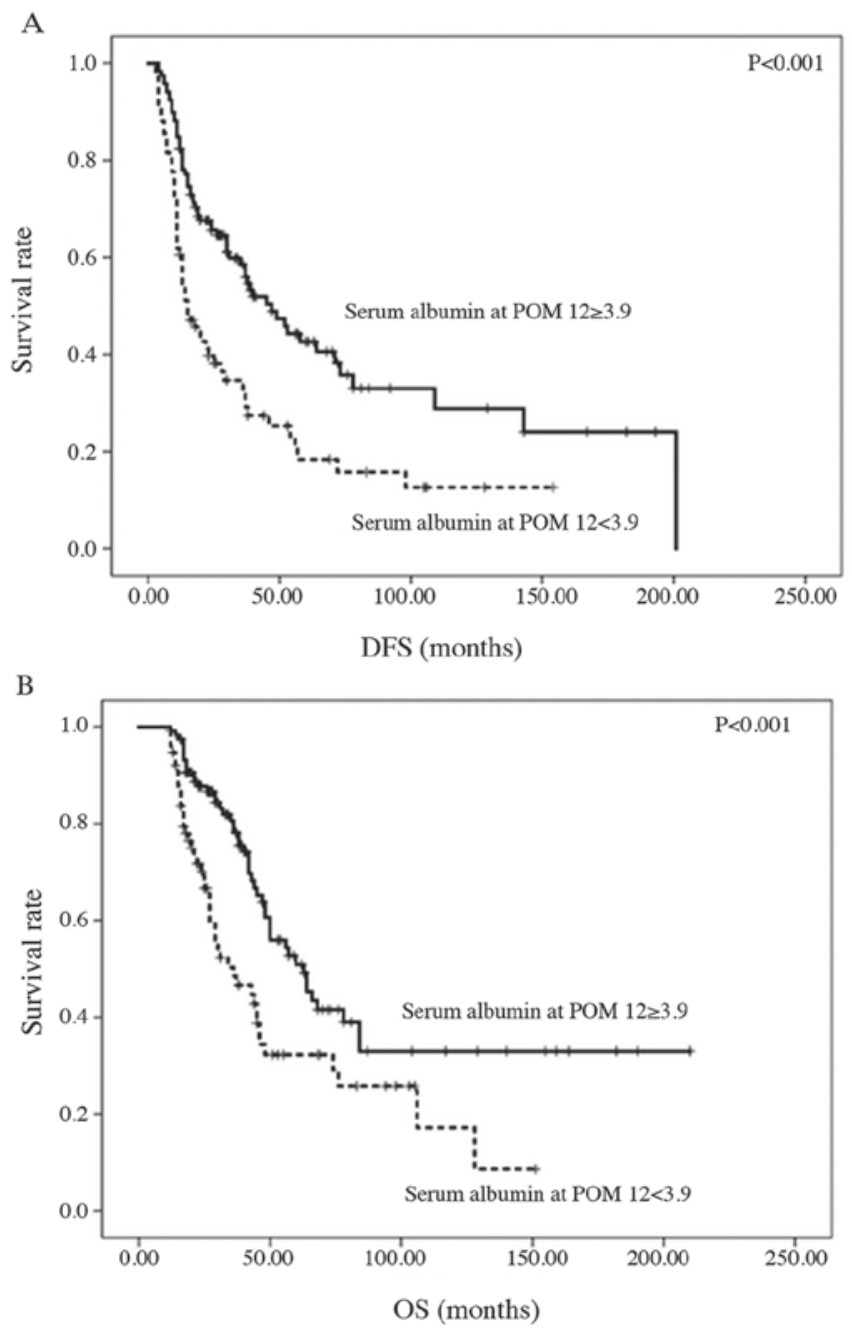

Figure 2. Relationship between postoperative serum albumin at POM 12 and (A) postoperative DFS and (B) postoperative OS. POM, postoperative month; DFS, disease-free survival; OS, overall survival.

decrease in the body strength (6). A postoperative lower serum albumin or insufficient albumin recovery may represent the metabolic changes in pancreatic cancer after surgery, and just against pancreatic cancer which is one of the most lethal and aggressive forms of cancer, serum albumin may be a potential biomarker for predicting earlier recurrence as well as tumor markers $(19,20)$. Matsuda et al $(21)$, reported that insufficient albumin recovery at POM 3 correlated with a poor prognosis in patients with esophageal cancer who had undergone transthoracic esophagectomy. In our study of patients with PDAC, the serum albumin recovery rate at POM 3 was predictive of DFS. Moreover, patients with a postoperative serum albumin recovery rate of $\geq 1.00$ at POM 3 had a longer DFS. Insufficient albumin recovery correlates with a poor prognosis in patients with various types of cancer.

A number of overlapping factors may result in postoperative hypoalbuminemia. It is unclear whether improving preoperative serum albumin levels leads to a favorable prognosis. However, considering these factors, we should endeavor to intervene after surgery. Early nutritional support contributes to patient survival (22-24). In the review article reported by Gilliland et al (25), they recommended that pancreatic
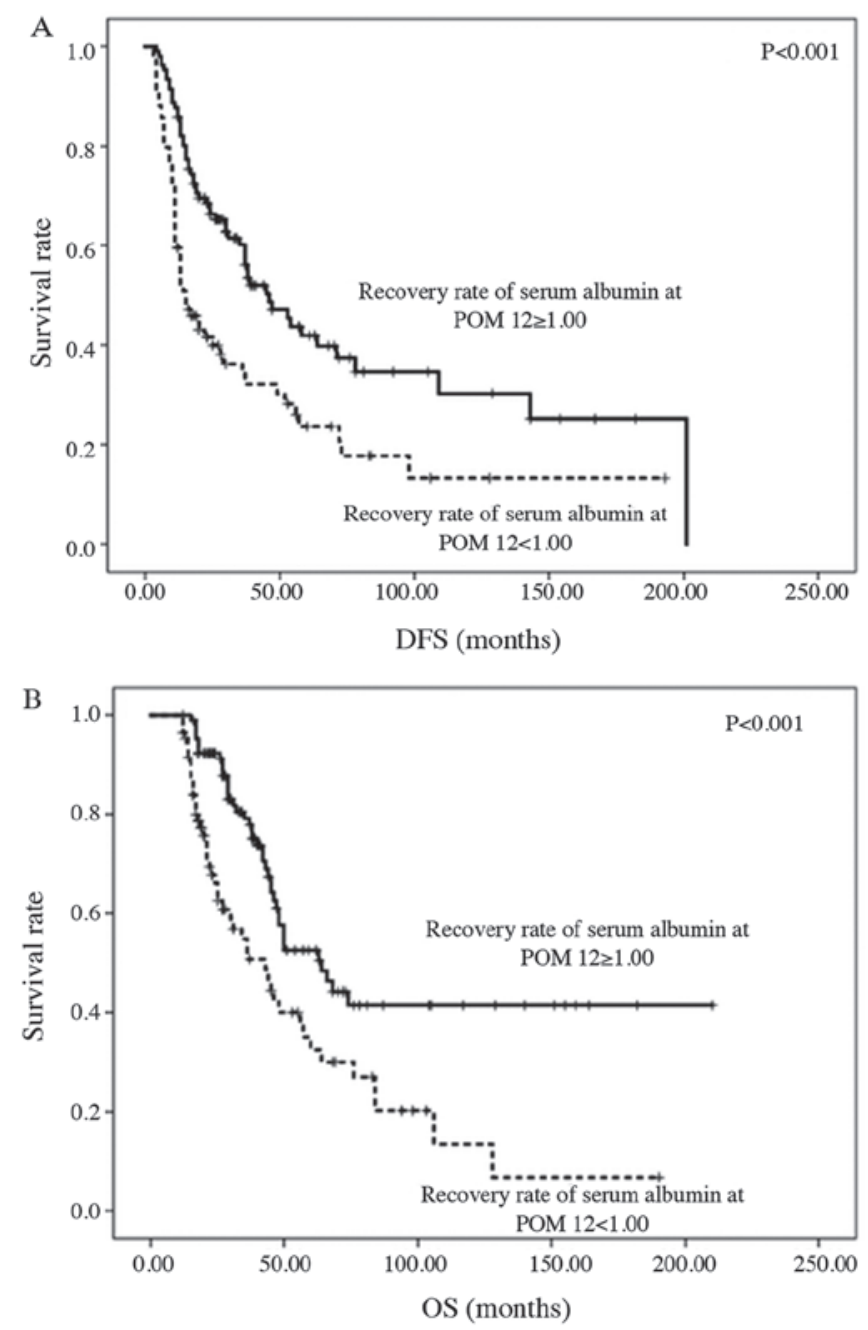

Figure 3. Relationship between the postoperative recovery rate of serum albumin at POM 12 and (A) postoperative DFS and (B) postoperative OS. POM, postoperative month; DFS, disease-free survival; OS, overall survival.

cancer patients with moderately decreased albumin levels $(<3.0 \mathrm{mg} / \mathrm{dl})$ or weight loss $>5 \%$ should still receive some form of nutritional supplementation prior to surgery, or feeding jejunostomy tubes intraoperatively to avoid undesirable patient outcomes associated with an insufficient nutritional intervention. Shu et al (26), suggested in their meta-analysis that postoperative early enteral nutrition (EEN) for patients with digestive tract surgery improves the nutritional status, promotes the functional recovery of the digestive system and reduces the risk of postoperative complications; they also reported a significant higher level of postoperative serum albumin in patients with ENN than those without EEN. It may be more effective to insert an enteral tube after surgery as early nutritional support for patients with poor nutritional status or with lower albumin level before surgery. However, it is unclear whether postoperative EEN influences long-term prognosis of PDAC, such as DFS or OS; thus, further studies are needed.

Okada et al (27), reported that a good nutritional status during chemotherapy is closely associated with the occurrence of adverse events and chemotherapy response to FOLFOX/FIRI therapy in patients with colorectal cancer. It prolonged DFS and OS in response to FOLFOX/FIRI 


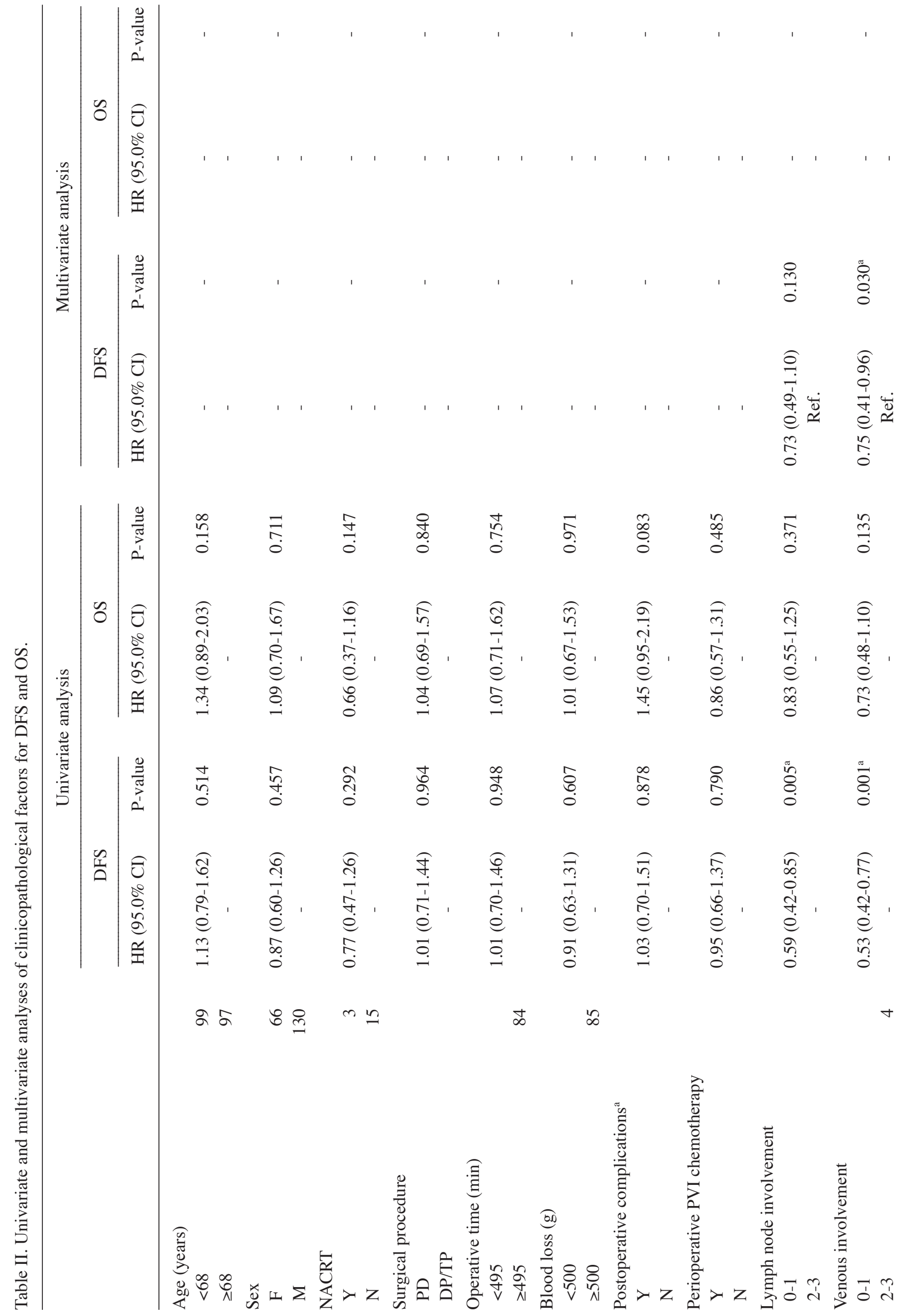




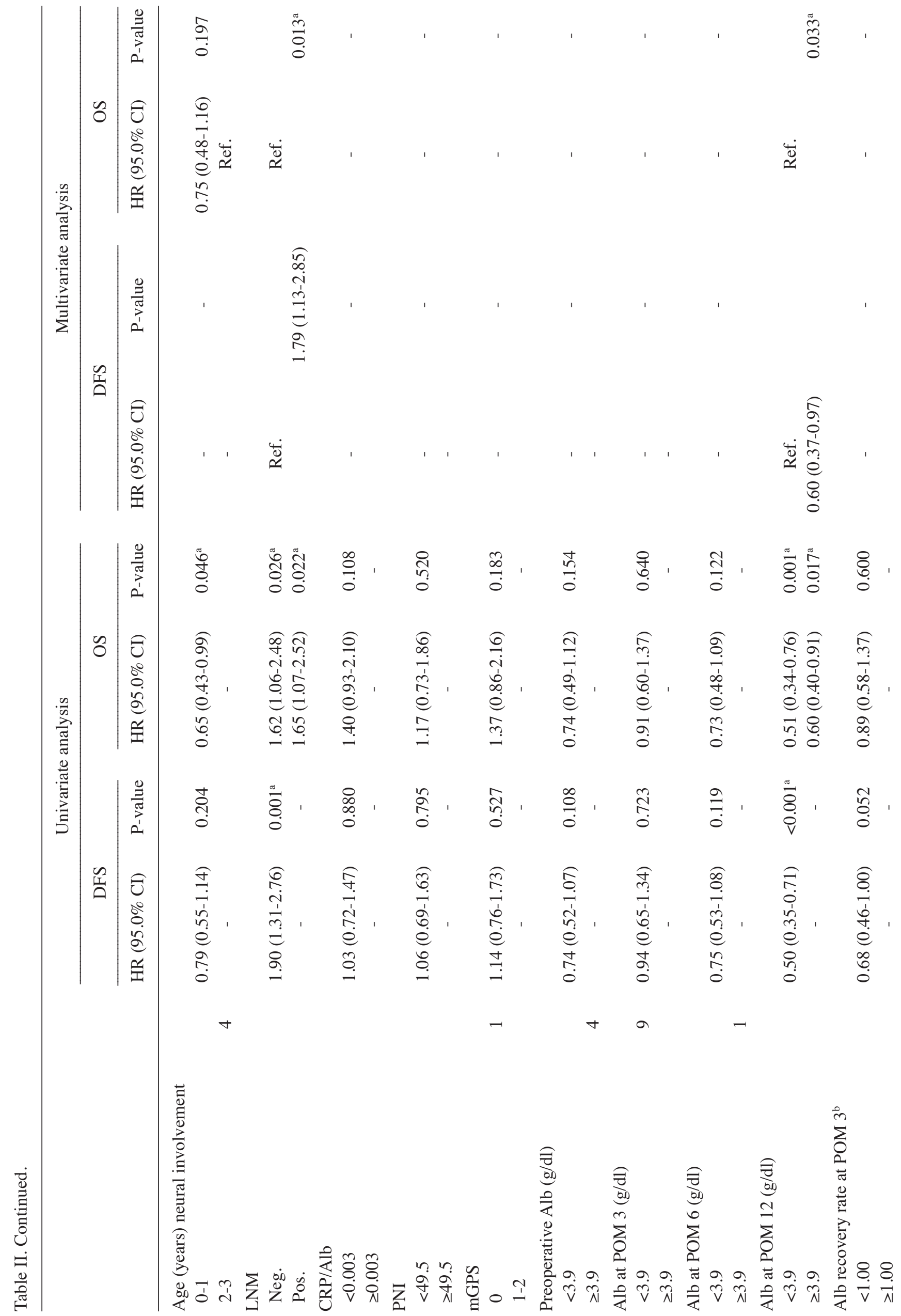


therapy. In this study, patients with a serum albumin level of $<3.9 \mathrm{~g} / \mathrm{dl}$ at POMs 6 and $12 \mathrm{did}$ not receive postoperative adjuvant chemotherapy compared to those with a serum albumin level of $\geq 3.9 \mathrm{~g} / \mathrm{dl}$ at POMs 6 and 12 . These patients also exhibited significantly early recurrence (within 1 year). Maintaining a good nutritional status during adjuvant chemotherapy may contribute to a higher response to cancer. Nutritional support is an important intervention for patients who are poorly nourished (e.g, with highly advanced cancer, such as PDAC).

According to JASPAC-01 (3), which is one of the randomized controlled trials of adjuvant chemoradiotherapy, we have typically administrated S-1 as adjuvant chemotherapy, which is repeated every 6 weeks for up to four cycles since 2014, and before 2014, we have mainly administrated gemcitabine as an adjuvant chemotherapy. We have guessed that the patients with lower serum albumin levels or insufficient albumin recovery at POM 12 tend to have lower serum albumin levels or insufficient albumin recovery at POM 6. Therefore, at POM 6, when adjuvant chemotherapy is finished, if the patients have a lower serum albumin level, insufficient albumin recovery, or high level of tumor markers associated with PDAC, such as CA19-9, we should consider a continuation of $\mathrm{S}-1$ as adjuvant chemotherapy or a different administration, such as mFOLFILINOX, which has recently been reported by Conroy et al (28), in a recent randomized control trial of the adjuvant chemotherapy, 'PRODIGE24/CCTGPA'.

Reduced serum albumin levels after surgical trauma are also associated with systemic inflammation (29). Reduced postoperative serum albumin levels are a marker of the stress response (30). Therefore, reducing postoperative surgical stress may result in the optimal early recovery of postoperative serum albumin levels. Anti-inflammatory drugs may be effective for inhibiting inflammatory mediators. Methylprednisolone treatment is associated with reduced interleukin- 6 and interleukin- 8 inflammatory cytokine levels during esophagectomy for esophageal carcinoma (31). Moreover, Takata et al (32) reported that postoperative ghrelin administration was effective for improving the postoperative clinical course of patients with esophageal cancer. However, there have been no reports of the benefits of anti-inflammatory drug use during pancreatectomy. Therefore, further research is needed.

This study has several limitations. First, this was a retrospective study conducted at a single institution with a relatively small number of patients. The prognostic significance of postoperative serum albumin levels has not been verified in a validation cohort. Second, the timing of serum albumin measurements had not been established because this study was retrospective. In our institution, patients were generally followed-up 1, 3, 6, and 12 months after surgery. Therefore, the timing of postoperative serum albumin measurements was almost the same, although there were patients who did not have blood tests at POMs 3,6, and 12. Although some hematological data were missing, the numbers were small and assumed to be random. Therefore, even if the patients with missing data were excluded, the outcomes of this study would not change. However, further prospective studies are needed to confirm these preliminary findings. 
In conclusion, the postoperative level and recovery rate of serum albumin are potential biomarkers for predicting the prognosis of patients with PDAC who have undergone curative resection. Further studies are needed to investigate the survival benefit of increasing postoperative serum albumin levels in these patients.

\section{Acknowledgements}

Not applicable.

\section{Funding}

No funding was received.

\section{Availability of data and materials}

The datasets used and analyzed during this study are available from the corresponding author on reasonable request.

\section{Authors' contributions}

YN, MK, and YK conceived and designed the study. YN drafted the manuscript. MS, HY, YA, KT, GO, AT and YE analyzed the data and critically revised the manuscript. All authors were involved in data interpretation and drafting the manuscript and have read and approved the final version of the manuscript.

\section{Ethics approval and consent to participate}

This study was conducted in accordance with the Declaration of Helsinki and local ethical legislation. The present study was approved by the Ethics Committee of Keio University School of Medicine. Due to the retrospective nature of the study, instead of obtaining informed consent from each patient, participants were given the opportunity to opt-out.

\section{Patient consent for publication}

Not applicable.

\section{Competing interests}

The authors declare that they have no competing interests.

\section{References}

1. Egawa S, Toma H, Ohigashi H, Okusaka T, Nakano A, Hatori T, Maguchi H, Yanagisawa A and Tanaka M: Japan pancreatic cancer registry; 30 th year anniversary: Japan pancreas society. Pancreas 41: 985-992, 2012.

2. Jemal A, Siegel R, Xu J and Ward E: Cancer statistics, 2010. CA Cancer J Clin 60: 277-300, 2010.

3. Verma V, Li J and Lin C: Neoadjuvant therapy for pancreatic cancer: Systematic review of postoperative morbidity, mortality, and complications. Am J Clin Oncol 39: 302-313, 2016.

4. UesakaK,BokuN,FukutomiA,Okamura Y,KonishiM,MatsumotoI, Kaneoka Y, Shimizu Y, Nakamori S, Sakamoto H, et al: Adjuvant chemotherapy of S-1 versus gemcitabine for resected pancreatic cancer: A phase 3, open-label, randomised, non-inferiority trial (JASPAC 01). Lancet 388: 248-257, 2016.

5. Farrugia A: Albumin usage in clinical medicine: Tradition or therapeutic? Transfus Med Rev 24: 53-63, 2010.
6. Gabay C and Kushner I: Acute-phase proteins and other systemic responses to inflammation. N Eng J Med 340: 448-454, 1999.

7. Namendys-Silva SA, Gonzalez-HerreraMO,Texcocano-Becerra J and Herrera-Gomez A: Hypoalbuminemia in critically ill patients with cancer: Incidence and mortality. Am J Hosp Palliat Care 28: 253-257, 2011.

8. Fuhrman MP: The albumin-nutrition connection: Separating myth from fact. Nutrition 18: 199-200, 2002.

9. Visser M, Kritchevsky SB, Newman AB, Goodpaster BH, Tylavsky FA, Nevitt MC and Harris TB: Lower serum albumin concentration and change in muscle mass: The health, aging and body composition study. Am J Clin Nutr 82: 531-537, 2005.

10. Haruki K, Shiba H, Shirai Y, Horiuchi T, Iwase R, Fujiwara Y, Furukawa K, Misawa T and Yanaga K: The C-reactive protein to albumin ratio predicts long-term outcomes in patients with pancreatic cancer after pancreatic resection. World J Surg 40: 2254-2260, 2016.

11. Kanda M, Fujii T, Kodera Y, Nagai S, Takeda S and Nakao A: Nutritional predictors of postoperative outcome in pancreatic cancer. Br J Surg 98: 268-274, 2011.

12. Imaoka H, Mizuno N, Hara K, Hijioka M, Tajika M, Tanaka T, Ishihara M, Yogi T, Tsutsumi H, Fujiyoshi T, et al: Evaluation of modified glasgow prognostic score for pancreatic cancer: A retrospective cohort study. Pancreas 45: 211-217, 2016.

13. Nakano Y, Kitago M, Matsuda S, Nakamura Y, Fujita Y, Imai S, Shinoda M, Yagi H, Abe Y, Hibi T, et al: KRAS mutations in cell-free DNA from preoperative and postoperative sera as a pancreatic cancer marker: A retrospective study. Br J Cancer 118: 662-669, 2018.

14. Nakano Y, Kitago M, Shinoda M, Abe Y, Yagi H, Hibi T, Takeuchi A, Aiura K, Itano O and Kitagawa Y: Clinical predictive factors of long-term survival after curative resection of pancreatic cancer: A retrospective study. Br J Cancer 6: 2278-2281, 2018.

15. Fujii-Nishimura Y, Nishiyama R, Kitago M, Masugi Y, Ueno A, Aiura K, Kawachi S, Kawaida M, Abe Y, Shinoda M, et al: Two cases of pathological complete response to neoadjuvant chemoradiation therapy in pancreatic cancer. Keio J Med 64: 26-31, 2015.

16. Japan Pancreas Society: Classification of pancreatic carcinoma. 2nd English ed. Tokyo: Kanehara, 2009.

17. Takahashi S, Aiura K, Saitoh J, Hayatsu S, Kitajima M and Ogata Y: Treatment strategy for pancreatic head cancer: Pylorus-preserving pancreatoduodenectomy, intraoperative radiotherapy and portal catheterization. Digestion 60 (Suppl): S130-S134, 1999.

18. Aiura K, Takahashi S, Matsui J, Ueda M and Kitagawa Y: Beneficial effects of 5-Fluorouracil and heparin-based portal infusion chemotherapy combined with mitomycin $\mathrm{C}$ and cisplatin after curative resection of pancreatic cancer. Pancreatology 10: 250-258, 2017.

19. McMillan DC, Watson WS, O'Gorman P, Tom Preston, Scott HR and McArdle CS: Albumin concentrations are primarily determined by the body cell mass and the systemic inflammatory response in cancer patients with weight loss. Nutr Cancer 39:210-213, 2001.

20. Schmid I, Schmitt M, Streiter M, Meilbeck R, Haas RJ and Stachel DK: Effects of soluble TNF receptor II (sTNF-RII), IL-1 receptor antagonist (IL-1 ra), tumor load and hypermetabolism on malnutrition in children with acute leukemia. Eur J Med Res 10: 457-461, 2005.

21. Matsuda S, Niihara M, Tsubosa Y, Sato H, Takebayashi K, Kawamorita K, Mori K, Tsushima T, Yasui H, Takeuchi H and Kitagawa Y: Clinical significance of postoperative recovery of serum albumin levels in patients with esophageal cancer who underwent transthoracic esophagectomy. Surg Today 46: 1138-1145, 2016.

22. Ravasco P, Monteiro-Grillo I, Vidal PM and Camilo ME: Dietary counseling improves patient outcomes: A prospective, randomized, controlled trial in colorectal cancer patients undergoing radiotherapy. J Clin Oncol 23: 1431-1438, 2005.

23. Roxburgh CSD, Crozier JEM, Maxwell F, Foulis AK, Brown J, McKee RF, Anderson JH, Horgan PG and McMillan DC: Comparison of tumour based (Petersen Index) and inflammation-based (Glasgow Prognostic Score) scoring systems in patients undergoing curative resection for colon cancer. $\mathrm{Br}$ J Cancer 100: 701-706, 2009.

24. McMillan DC, Crozier JE, Canna K, Angerson WJ and McArdle CS: Evaluation of an inflammation based prognostic score (GPS) in patients undergoing resection for colon and rectal cancer. Int J Colorectal Dis 22: 881-886, 2007. 
25. Gilliland TM, Villafane-Ferriol N, Shah KP, Shah RM Tran Cao HS, Massarweh NN, Silberfein EJ, Choi EA, Hsu C and McElhany CA: Nutritional and metabolic derangements in pancreatic cancer and pancreatic resection. Nutrients 9: E243, 2017.

26. Shu XL, Kang K, Gu LJ and Zhang YS: Effect of early enteral nutrition on patients with digestive tract surgery: A meta-analysis of randomized controlled trials. Exp Ther Med 12: 2136-2144, 2016.

27. Okada S, Yamazaki S, Kaiga T, Funada T, Kochi M and Takayama T: Impact of nutritional status in the era of FOLFOX/FIRI-based chemotherapy, World J Surg Oncol 15: $162-169,2017$.

28. Conroy T, Hammel P, Hebbar M, Ben Abdelghani M, Wei AC, Raoul JL, Chone L, Francois E, Artru A, Biagi JJ, et al: Folfirinox or gemcitabine as adjuvant therapy for pancreatic cancer. $\mathrm{N}$ Engl J Med 379: 2395-2406, 2018
29. Fleck A, Raines G, Hawker F, Trotter J, Wallace PI, Ledingham IM and Calman KC: Increased vascular permeability: A major cause of hypoalbuminaemia in disease and injury. Lancet 1: 781-784, 1985.

30. Hubner M, Mantziari S, Demartines N, Pralong F, Coti-Bertrand P and Schafer M: Postoperative albumin drop is a marker for surgical stress and a predictor for clinical outcome: A pilot study. Gastroenterol Res Pract : 8743187, 2016.

31. Gao Q, Mok HP, Wang WP, Xiao F and Chen LQ: Effect of perioperative glucocorticoid administration on postoperative complications following esophagectomy: A meta-analysis. Oncol Lett 7: 349-356, 2014.

32. Takata A, Takiguchi S, Miyazaki Y, Miyata H, Takahashi T, Kurokawa Y, Yamasaki M, Nakajima K, Mori M, Kanagawa K and Doki Y: Randomized phase II study of the anti-inflammatory effect of ghrelin during the postoperative period of esophagectomy. Ann Surg 262: 230-236, 2015. 